\title{
ANTI-WINDUP DESIGN WITH GUARANTEED REGIONS OF STABILITY FOR DISCRETE-TIME LINEAR SYSTEMS WITH SATURATING CONTROLS
}

\author{
João Manoel Gomes da Silva Jr.* \\ jmgomesdeletro.ufrgs.br
}

\author{
Romeu Reginatto* \\ romeudeletro.ufrgs.br
}

\author{
Sophie Tarbouriech ${ }^{\dagger}$ \\ tarbourelaas.fr
}

*UFRGS - Departamento de Engenharia Elétrica, Av. Osvaldo Aranha 103, 90035-190 Porto Alegre-RS, Brazil.

${ }^{\dagger}$ LAAS-CNRS, 7 Avenue du Colonel Roche, 31077 Toulouse cedex 4, France.

\begin{abstract}
The purpose of this paper is to study the determination of stability regions for discrete-time linear systems with saturating controls through anti-windup schemes. Considering that a linear dynamic output feedback has been designed to stabilize the linear discrete-time system (without saturation), a method is proposed for designing an anti-windup gain that maximizes an estimate of the basin of attraction of the closed-loop system in the presence of saturation. It is shown that the closed-loop system obtained from the controller plus the anti-windup gain can be modeled by a linear system connected to a deadzone nonlinearity. From this model, stability conditions based on quadratic Lyapunov functions are stated. Algorithms based on LMI schemes are proposed for computing both the anti-windup gain and an associated stability region.
\end{abstract}

KEYWORDS: Anti-windup, control saturation, discrete-time systems, regions of stability.

\section{RESUMO}

Este artigo tem por objetivo o estudo da determinação de regiões de estabilidade para sistemas lineares discretos no tempo com controles saturantes, através da utilização de la-

\footnotetext{
Artigo submetido em 05/12/02

1a. Revisão em 27/05/03; 2a. Revisão em 21/07/03

Aceito sob recomendação do Ed. Assoc. Prof. José R. C. Piqueira
}

ços de anti-windup. Considerando que um compensador dinâmico de saída é previamente projetado para estabilizar o sistema linear em tempo discreto (i.e. desconsiderando-se a saturação), é proposto um método para projetar um ganho de anti-windup que maximize a região de atração do sistema em malha fechada na presença de saturação. É mostrado que o sistema em malha fechada, obtido a partir do controlador com o termo de anti-windup, pode ser modelado por um sistema linear em cascata com uma não-linearidade do tipo zona-morta. A partir deste modelamento, condições de estabilidade baseadas em funções de Lyapunov quadráticas são estabelecidas. Algortimos baseados na solução de LMIs são propostos para computar simultaneamente o ganho de antiwindup e a região de estabilidade associada.

PALAVRAS-CHAVE: Anti-windup, saturação de controle, sistemas discretos no tempo, regiões de establidade.

\section{INTRODUCTION}

The basic idea underlining anti-windup designs for linear systems with saturating actuators is to introduce control modifications in order to recover, as much as possible, the performance induced by a previous design carried out on the basis of the unsaturated system. First results on anti-windup consisted on ad-hoc methods intended to work with standard PID controllers (Fertik and Ross, 1967; Åström and Rundqwist, 1989) which are commonly used in present commercial controllers. Nonetheless, major improvements in 
this field have been achieved in the last decade as it can be observed in (Barbu et al., 2000; Kothare and Morari, 1999; Teel, 1999; Burgat and Tarbouriech, 1998; Kapoor et al., 1998; Teel and Kapoor, 1997; Kothare and Morari, 1997; Miyamoto and Vinnicombe, 1996) among others.

Several results on the anti-windup problem are concerned with achieving global stability properties. Since global results cannot be achieved for open-loop unstable linear systems in the presence of actuator saturation, local results have to be developed. In this context, a key issue is the determination of domains of stability for the closed-loop system (estimates of the regions of attraction). With very few exceptions, most of the local results available in the literature of anti-windup do not provide explicit characterization of the domain of stability.

In (Gomes da Silva Jr. et al., 2002) an attempt has been made to fill in this gap by providing two design algorithms that explicitly optimize a criterion aiming at maximizing a stability domain of the closed-loop system. The results have been provided as LMI problems and covered continuous-time linear systems with saturating actuators.

In this paper we further fill in such a gap by providing similar results for discrete-time linear system with saturating actuators. For a given linear output feedback design that yields a certain performance when in closed-loop with the unsaturated linear system, an anti-windup gain is designed in order to enlarge the region of asymptotic stability of the closedloop system. Results are stated in terms of LMI problems derived from quadratic stability design criteria.

The anti-windup problem for discrete-time systems has received less attention in the literature. It has been addressed in (Fertik and Ross, 1967; Walgama and Sternby, 1993) (see references therein), in the scope of the conditioning technique, and in (Shamma, 1999) in the context of constrained regulation. Similarly as in the continuous time case, the proposed designs do not explicitly address the problem of enlarging the domain of stability of the closed-loop system. This point is the central issue of this paper.

The paper is organized as follows. In section 2 we state the problem being considered and provide the main definitions and concepts required in the paper. Stability conditions for the closed-loop systems are provided in section 3 by employing quadratic Lyapunov functions. Based on the results of section 3, numerical algorithms to synthesize the anti-windup gain are developed in section 4 . Section 5 provides simulation results for a case study, illustrating the effectiveness of the proposed design technique. Concluding remarks are given in section 6.

Notations. For any vector $x \in \Re^{n}, x \succeq 0$ means that all the components of $x$, denoted $x_{(i)}$, are nonnegative. For two vectors $x, y$ of $\Re^{n}$, the notation $x \succeq y$ means that $x_{(i)}-$ $y_{(i)} \geq 0, \forall i=1, \ldots, n$. The elements of a matrix $A \in$ $\Re^{m * n}$ are denoted by $A_{(i, j)}, \quad i=1, \ldots, m, j=1, \ldots, n$. $A_{(i)}$ denotes the $i$ th row of matrix $A$. For two symmetric matrices, $A$ and $B, A>B$ means that $A-B$ is positive definite. $A^{\prime}$ denotes the transpose of $A$. $\operatorname{diag}(x)$ denotes a diagonal matrix obtained from vector $x . I_{m}$ denotes the $m$ order identity matrix. $C o\{\cdot\}$ denotes a convex hull.

\section{PROBLEM STATEMENT}

Consider the discrete-time linear system

$$
\begin{cases}x(t+1) & =A x(t)+B u(t) \\ y(t) & =C x(t)\end{cases}
$$

where $x(t) \in \Re^{n}, u(t) \in \Re^{m}, y(t) \in \Re^{p}$ are the state, the input and the measured output vectors, respectively, and $t \in \mathcal{N}$. Matrices $A, B$ and $C$ are real constant matrices of appropriate dimensions. We suppose that system (1) satisfies the following assumptions.

Assumption 1 Pairs $(A, B)$ and $(C, A)$ are assumed to be controllable and observable respectively.

Assumption 2 The input vector $u$ is subject to amplitude limitations defined as follows:

$$
\mathcal{U}_{0}=\left\{u \in \Re^{m} ;-u_{0} \preceq u \preceq u_{0}\right\}
$$

where $u_{0(i)}>0, i=1, \ldots, m$ denote the control amplitude bounds.

Considering system (1) and the assumptions above, we assume that an $n_{c}$-order dynamic output stabilizing compensator

$$
\begin{aligned}
x_{c}(t+1) & =A_{c} x_{c}(t)+B_{c} y(t) \\
v_{c}(t) & =C_{c} x_{c}(t)+D_{c} y(t)
\end{aligned}
$$

where $x_{c}(t) \in \Re^{n_{c}}$ is the controller state, $u_{c}=y(t)$ is the controller input and $v_{c}(t)$ is the controller output, was designed to guarantee some performance requirements and the stability of the closed-loop system in the absence of control saturation. In consequence of the control bounds, the actual control signal to be injected in the system is a saturated one, that is,

$$
u(t)=\operatorname{sat}\left(v_{c}(t)\right)=\operatorname{sat}\left(C_{c} x_{c}(t)+D_{c} C x(t)\right)
$$

where each component of $\operatorname{sat}\left(v_{c}(t)\right)$ is defined, $\forall i=$ $1, \ldots, m$, by:

$$
\operatorname{sat}\left(v_{c}\right)_{(i)} \triangleq\left\{\begin{array}{cl}
-u_{0(i)} & \text { if } v_{c(i)}(t)<-u_{0(i)} \\
v_{c(i)}(t) & \text { if }-u_{0(i)} \leq v_{c(i)}(t) \leq u_{0(i)} \\
u_{0(i)} & \text { if } v_{c(i)}(t)>u_{0(i)}
\end{array}\right.
$$


In order to mitigate the undesirable effects of the windup, caused by input saturation, an anti-windup term $E_{c}\left(\operatorname{sat}\left(v_{c}(t)\right)-v_{c}(t)\right)$ can be added to the controller (Åström and Rundqwist, 1989; Teel, 1999). Thus, considering the dynamic controller and this anti-windup strategy, the closedloop system reads:

$$
\begin{aligned}
x(t+1)= & A x(t)+B s a t\left(v_{c}(t)\right) \\
y(t)= & C x(t) \\
x_{c}(t+1)= & A_{c} x_{c}(t)+B_{c} y(t) \\
& \quad+E_{c}\left(\operatorname{sat}\left(v_{c}(t)\right)-v_{c}(t)\right) \\
v_{c}(t)= & C_{c} x_{c}(t)+D_{c} y(t)
\end{aligned}
$$

Define now an extended state vector

$$
\xi(t)=\left[\begin{array}{c}
x(t) \\
x_{c}(t)
\end{array}\right] \in \Re^{n+n_{c}}
$$

and the following matrices

$$
\begin{gathered}
\mathbf{A}=\left[\begin{array}{cc}
A+B D_{c} C & B C_{c} \\
B_{c} C & A_{c}
\end{array}\right] ; \mathbf{B}=\left[\begin{array}{l}
B \\
0
\end{array}\right] \\
\mathbf{R}=\left[\begin{array}{c}
0 \\
I_{n_{c}}
\end{array}\right] ; \mathbf{K}=\left[\begin{array}{ll}
D_{c} C & C_{c}
\end{array}\right]
\end{gathered}
$$

Hence, from (6) and (5), the closed-loop system reads:

$$
\xi(t+1)=\mathbf{A} \xi(t)-\left(\mathbf{B}+\mathbf{R} E_{c}\right) \psi(\mathbf{K} \xi(t))
$$

with

$$
\psi(\mathbf{K} \xi(t))=v_{c}(t)-\operatorname{sat}\left(v_{c}(t)\right)=\mathbf{K} \xi(t)-\operatorname{sat}(\mathbf{K} \xi(t))
$$

The matrix $\mathbf{A}$ is supposed to be Hurwitz, i.e., in the absence of control bounds, the closed-loop system would be globally stable.

By definition,

$$
\psi(\mathbf{K} \xi)=\left[\begin{array}{lll}
\psi\left(\mathbf{K}_{(1)} \xi\right) & \ldots & \psi\left(\mathbf{K}_{(m)} \xi\right)
\end{array}\right]^{\prime}
$$

is a decentralized deadzone nonlinearity and satisfies the following sector condition (see figure 1) (Khalil., 1992), (Gomes da Silva Jr. et al., 2001):

$$
\psi(\mathbf{K} \xi)^{\prime}[\psi(\mathbf{K} \xi)-\Lambda \mathbf{K} \xi] \leq 0, \quad \forall \xi \in S\left(\mathbf{K}, u_{0}^{\lambda}\right)
$$

where $\Lambda \in \Re^{m * m}$ is a positive diagonal matrix and the set $S\left(\mathbf{K}, u_{0}^{\lambda}\right)$ is a polyhedral set defined as follows:

$$
S\left(\mathbf{K}, u_{0}^{\lambda}\right)=\left\{\xi \in \Re^{n+n_{c}} ;-u_{0}^{\lambda} \preceq \mathbf{K} \xi \preceq u_{0}^{\lambda}\right\}
$$

where $u_{0(i)}^{\lambda} \triangleq \frac{u_{0(i)}}{1-\Lambda_{(i, i)}}, i=1, \ldots, m$.

The basin of attraction of system (7) is defined as the set of all $\xi \in \Re^{n+n_{c}}$ such that for $\xi(0)=\xi$ the corresponding trajectory converges asymptotically to the origin. In particular,

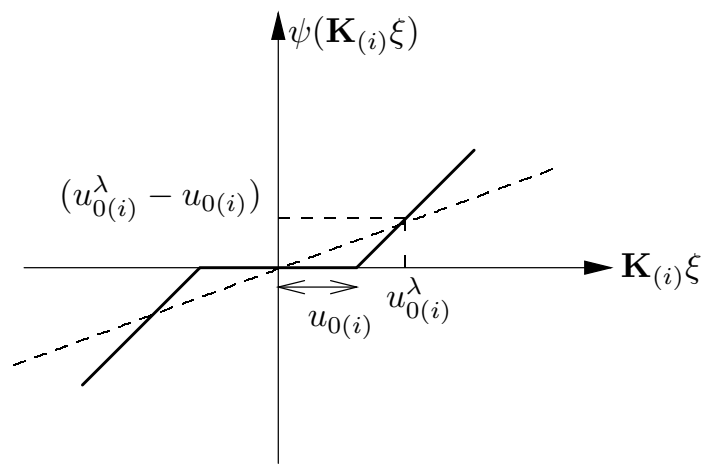

Figure 1: function $\psi(\mathbf{K} \xi)$

when the global stability of the system is ensured the basin of attraction corresponds to the whole state space. However, in the general case, the exact characterization of the basin of attraction is not possible. In this case, it is important to obtain estimates of the basin of attraction. Consider then the following definition:

Definition 1 A set $\mathcal{E}$ is said to be a region of asymptotic stability for the system (7) if for all $\xi(0) \in \mathcal{E}$ the corresponding trajectory converges asymptotically to the origin

Hence, the idea is to use regions of stability in order to approximate the basin of attraction (Khalil., 1992).

The problem we aim to solve throughout this paper is summarized as follows.

Problem 1 Determine the anti-windup gain matrix $E_{c}$ and an associated region of asymptotic stability, as large as possible, for the closed-loop system (7).

Of course, the implicit objective in Problem 1 is to optimize the size of the basin of attraction for the closed-loop system (7) over the choice of the gain matrix $E_{c}$. This can be accomplished indirectly by searching for an anti-windup gain $E_{c}$ that leads to a region of stability for the closed-loop system as large as possible.

In order to address Problem 1, we propose to use quadratic Lyapunov functions and ellipsoidal regions of stability, as will be see in the sequel.

Remark 1 The region of linear behavior of system (7) is defined as follows

$$
R_{L}=\left\{\xi \in \Re^{n+n_{c}} ;-u_{0} \preceq \mathbf{K} \xi \preceq u_{0}\right\}
$$

Note that inside $R_{L}$ there is no saturation occurrence, i.e. $\psi(\mathbf{K} \xi(t))=0$. 


\section{STABILITY CONDITIONS}

Throughout this section, we consider as Lyapunov candidate function, the quadratic function:

$$
V(\xi(t))=\xi(t)^{\prime} P \xi(t), P=P^{\prime}>0
$$

Proposition 1 If there exist a symmetric positive definite matrix $W \in \Re^{\left(n+n_{c}\right) \times\left(n+n_{c}\right)}$, diagonal positive definite matrices $\Lambda \in \Re^{m \times m}$ and $S \in \Re^{m \times m}$, a matrix $Z \in \Re^{n_{c} \times m}$ and a positive scalar $\gamma$ satisfying:

$$
\begin{gathered}
{\left[\begin{array}{ccc}
W & -W \mathbf{K}^{\prime} \Lambda & -W \mathbf{A}^{\prime} \\
-\Lambda \mathbf{K} W & 2 S & S \mathbf{B}^{\prime}+Z^{\prime} \mathbf{R}^{\prime} \\
-\mathbf{A} W & \mathbf{B} S+\mathbf{R} Z & W
\end{array}\right]>0} \\
{\left[\begin{array}{cc}
W & \left(1-\Lambda_{(i, i)}\right) W \mathbf{K}_{(i)}^{\prime} \\
\left(1-\Lambda_{(i, i)}\right) \mathbf{K}_{(i)} W & \gamma u_{0(i)}^{2}
\end{array}\right] \geq 0} \\
i=1, \ldots, m \\
0<\Lambda_{(i, i)} \leq 1, i=1, \ldots, m
\end{gathered}
$$

then for the gain matrix $E_{c}=Z S^{-1}$ the ellipsoid $\mathcal{E}\left(P, \gamma^{-1}\right)=\left\{\xi \in \Re^{n+n_{c}} ; \xi^{\prime} P \xi \leq \gamma^{-1}\right\}$, with $P=W^{-1}$, is a region of stability for the system (7)

Proof. The satisfaction of relations (14) and (15) implies that the set $\mathcal{E}\left(P, \gamma^{-1}\right)$ is included in the polyhedral set $S\left(\mathbf{K}, u_{0}^{\lambda}\right)$. Hence, for all $\xi(t) \in \mathcal{E}\left(P, \gamma^{-1}\right)$ it follows that $\psi(\mathbf{K} \xi(t))=$ $\mathbf{K} \xi(t)-\operatorname{sat}(\mathbf{K} \xi(t))$ satisfies the sector condition (9). By considering the quadratic Lyapunov function as defined in (12) and by computing the variation of $V(\xi(t))$ along the trajectories of system (7) one gets:

$$
\begin{aligned}
& \Delta V(\xi(t))=V(\xi(t))-V(\xi(t+1))= \\
& \quad=\xi(t)^{\prime} P \xi(t)-\xi(t)^{\prime}\left(\mathbf{A}^{\prime} P \mathbf{A}\right) \xi(t) \\
& \quad+2 \xi(t)^{\prime} \mathbf{A}^{\prime} P\left(\mathbf{B}+\mathbf{R} E_{c}\right) \psi(\mathbf{K} \xi(t)) \\
& \quad-\psi(\mathbf{K} \xi(t))^{\prime}\left(\mathbf{B}+\mathbf{R} E_{c}\right)^{\prime} P\left(\mathbf{B}+\mathbf{R} E_{c}\right) \psi(\mathbf{K} \xi(t))
\end{aligned}
$$

Thus, by using the sector condition (9) and the S-procedure with a positive definite diagonal matrix $T$ it follows ${ }^{1}$ :

$$
\begin{aligned}
& \Delta V(\xi(t)) \geq \quad \xi^{\prime} P \xi-\xi^{\prime}\left(\mathbf{A}^{\prime} P \mathbf{A}\right) \xi \\
& \quad+2 \xi^{\prime} \mathbf{A}^{\prime} P\left(\mathbf{B}+\mathbf{R} E_{c}\right) \psi \\
& \quad-\psi^{\prime}\left(\mathbf{B}+\mathbf{R} E_{c}\right)^{\prime} P\left(\mathbf{B}+\mathbf{R} E_{c}\right) \psi \\
& \quad+2 \sum_{i=1}^{m} T_{(i, i)} \psi_{(i)}^{\prime}\left[\psi_{(i)}-\Lambda_{(i, i)} \mathbf{K}_{(i)} \xi\right]
\end{aligned}
$$

or equivalently

$$
\Delta V(\xi(t)) \geq\left[\begin{array}{ll}
\xi^{\prime} & \psi^{\prime}
\end{array}\right]\left[\begin{array}{ll}
X_{1} & X_{2} \\
X_{2}^{\prime} & X_{3}
\end{array}\right]\left[\begin{array}{l}
\xi \\
\psi
\end{array}\right]
$$

\footnotetext{
${ }^{1}$ For notational simplicity we drop the time dependence and consider $\xi(t)=\xi$ and $\psi(\mathbf{K} \xi(t))=\psi$.
}

where

$$
\begin{aligned}
& X_{1}=P-\mathbf{A}^{\prime} P \mathbf{A} \\
& X_{2}=\mathbf{A}^{\prime} P\left(\mathbf{B}+\mathbf{R} E_{c}\right)-\mathbf{K}^{\prime} \Lambda T \\
& X_{3}=2 T-\left(\mathbf{B}+\mathbf{R} E_{c}\right)^{\prime} P\left(\mathbf{B}+\mathbf{R} E_{c}\right)
\end{aligned}
$$

Note now that, by Schur's complement, relation (13) is equivalent to

$$
\begin{aligned}
& {\left[\begin{array}{cc}
W & -W \mathbf{K}^{\prime} \Lambda \\
-\Lambda \mathbf{K} W & 2 S
\end{array}\right]-} \\
& {\left[\begin{array}{c}
-W \mathbf{A}^{\prime} \\
(\mathbf{B} S+\mathbf{R} Z)^{\prime}
\end{array}\right] P\left[\begin{array}{ll}
-\mathbf{A} W & (\mathbf{B} S+\mathbf{R} Z)]>0
\end{array}\right.}
\end{aligned}
$$

Considering now $T=S^{-1}$ and pre and post multiplying (19) by

$$
\left[\begin{array}{ll}
P & 0 \\
0 & T
\end{array}\right]
$$

it follows that

$$
\left[\begin{array}{ll}
X_{1} & X_{2} \\
X_{2}^{\prime} & X_{3}
\end{array}\right]>0
$$

As a result, the quadratic form in (18) is positive definite implying $\Delta V(\xi(t))>0$ (i.e $V(\xi(t+1)<V(\xi(t))$ ). Since this reasoning is valid $\forall \xi(t) \in \mathcal{E}\left(P, \gamma^{-1}\right), \xi(t) \neq 0$, it follows that the function $V(\xi(t))$ is strictly decreasing along the trajectories of system (7). Hence, we can conclude that $\mathcal{E}\left(P, \gamma^{-1}\right)$ is a stability region for system (7) which means that for any $\xi(0) \in \mathcal{E}\left(P, \gamma^{-1}\right)$, the corresponding trajectory converges asymptotically to the origin.

Proposition 1 gives a condition for the local stability of system (5) inside an ellipsoidal region in the state space. Note that, the larger are the $\Lambda_{(i, i)}$, the larger is the region $S\left(\mathbf{K}, u_{0}^{\lambda}\right)$ where the relation (17) can be verified and, as a consequence, a large contractive ellipsoid can be included. We can then state the following corollary, concerning a global stability condition.

Corollary 1 If there exist a symmetric positive definite matrix $W \in \Re^{\left(n+n_{c}\right) \times\left(n+n_{c}\right)}$, a diagonal positive definite matrix $S \in \Re^{m \times m}$ and a matrix $Z \in \Re^{n_{c} \times m}$ satisfying:

$$
\left[\begin{array}{ccc}
W & -W \mathbf{K}^{\prime} & -W \mathbf{A}^{\prime} \\
-\mathbf{K} W & 2 S & S \mathbf{B}^{\prime}+Z^{\prime} \mathbf{R}^{\prime} \\
-\mathbf{A} W & \mathbf{B} S+\mathbf{R} Z & W
\end{array}\right]>0
$$

then, for $E_{c}=Z S^{-1}$, system (7) is globally asymptotically stable.

Note that (21) corresponds to verify (13) with $\Lambda=I_{m}$. In this case, the nonlinearity $\psi(\mathbf{K} \xi)$ satisfies the sector condition $\forall \xi \in \Re^{n+n_{c}}$, i.e., the region $S\left(\mathbf{K}, u_{0}^{\lambda}\right)$ corresponds to the whole state space. 


\section{NUMERICAL ANTI-WINDUP GAIN DE- SIGN}

Based on the result stated in Proposition 1, in this section we aim to present some numerical algorithms in order to solve Problem 1. The main idea is to obtain an anti-windup gain matrix that ensures the local stability of the closed-loop system in a region of the state space $\Re^{n+n_{c}}$. We are then interested in one of the following cases:

1. A set of admissible initial conditions, $\Xi_{0} \subset \Re^{n+n_{c}}$, for which asymptotic stability must be ensured, is given.

2. We aim to design the anti-windup gain in order to maximize the estimation of the basin of attraction associated to it. In other words, we want to compute $E_{c}$ such that the associated region of asymptotic stability is as large as possible considering some size criterion.

Both cases can be addressed if we consider a set $\Xi_{0}$ with a given shape and a scaling factor $\beta$. For example, let $\Xi_{0}$ be defined as a polyhedral set described by its vertices:

$$
\Xi_{0} \triangleq C o\left\{v_{1}, v_{2}, \ldots, v_{r}\right\}
$$

where $v_{r} \in \Re^{n+n_{c}}, r=1, \ldots, n_{r}$. Note also that vectors $v_{i}$ can represent the directions for which the region of stability should be maximized.

Recalling Proposition 1, we aim at searching for matrices $W$, $\Lambda, S, Z$ and a scalar $\gamma$ in order to satisfy

$$
\beta \Xi_{0} \subset \mathcal{E}\left(P, \gamma^{-1}\right)
$$

In case 1 , mentioned above, this problem reduces to a feasibility problem with $\beta=1$ whereas in case 2 , the goal will be to maximize $\beta$. The problem of maximizing $\beta$ can be accomplished by solving the following optimization problem:

$$
\begin{aligned}
& \min _{W, Z, S, \Lambda, \mu, \gamma} \mu \\
& \text { subject to } \\
& \text { (i) }\left[\begin{array}{ccc}
W & -W \mathbf{K}^{\prime} \Lambda & -W \mathbf{A}^{\prime} \\
-\Lambda \mathbf{K} W & 2 S & S \mathbf{B}^{\prime}+Z^{\prime} \mathbf{R}^{\prime} \\
-\mathbf{A} W & \mathbf{B} S+\mathbf{R} Z & W
\end{array}\right]>0 \\
& \text { (ii) }\left[\begin{array}{cc}
W & \left(1-\Lambda_{(i, i)}\right) W \mathbf{K}_{(i)}^{\prime} \\
\left(1-\Lambda_{(i, i)}\right) \mathbf{K}_{(i)} W & \gamma u_{0(i)}^{2}
\end{array}\right] \geq 0, \\
& \text { (iii) }\left[\begin{array}{cc}
\gamma \mu & \gamma v_{r}^{\prime} \\
\gamma v_{r} & W
\end{array}\right] \geq 0 \quad r=1, \ldots, n_{v} \\
& \text { (iv) } \mu>0
\end{aligned}
$$

Considering $\beta=1 / \sqrt{\mu}$, the minimization of $\mu$ implies the maximization of $\beta$. The satisfaction of the inclusion relation (22) is ensured by the LMI (iii). Unfortunately, (23) is a
BMI problem in variables $W, \Lambda, \gamma$ and $\mu$. However, in the single-input case, since $\Lambda$ is a scalar, an optimal solution to the problem can easily be found by taking $\Lambda$ iteratively over a grid and solving (23) as an LMI problem. In this case, since $\Lambda$ is fixed, $\gamma$ can be considered as equal to 1 without loss of generality. This strategy can be pursued even in the case $m=2$, where a bidimensional grid must be considered and the optimal solution to problem (23) can be searched over the grid. For $m>2$, the grid strategy becomes more difficult to implement. In this case, the following algorithm, in which the basic idea is to iterate between steps where we fix $\Lambda$ or $W$, can be considered.

\section{Algorithm 1 :}

- Step 0: Initialization of $\Lambda$.

- Step 1: Fix $\Lambda$ obtained as solution of previous step and solve problem (23) with $\gamma=1$.

- Step 2: Fix $W$ obtained in step 1 and solve the following problem:

$$
\begin{gathered}
\min _{\Lambda, Z, S, \mu, \gamma} \mu \\
\text { subject to } \\
(i),(i i) \text { from }(23) \\
(\text { iii }) \mu W-v_{r} v_{r}^{\prime} \gamma \geq 0 \quad r=1, \ldots, n_{v} \\
(i v) \mu>0
\end{gathered}
$$

- Step 3: If the difference between $\mu$ obtained in step 2 and the one obtained in the previous iteration is greater than a desired accuracy go to step 1, otherwise set $E_{c}=$ $Z S^{-1}$ and stop.

In both steps, an eigenvalue problem is solved (Boyd et al., 1994) and the satisfaction of the inclusion relation (22) is ensured by the LMI (iii).

The idea behind step two in Algorithm 1 is the attempt of increasing the scalars $\lambda_{(i)}$, in order to increase the region $S\left(\mathbf{K}, u_{0}^{\lambda}\right)$ and, as a consequence, make it possible to include a larger ellipsoidal set associated to $W$. It is worth noticing that the optimal matrices obtained in step 1, and the fixed $\Lambda$, are a feasible solution for step 2 . Hence, in the worst case, the optimal solution of step 2 will be the same of the one in step 1. Conversely, the optimal matrices obtained as solution of step 2, considering $W$ normalized with respect to $\gamma$, consists in a feasible solution for step 1 . These facts ensures that, considering a reasonable numerical accuracy, the value of $\mu$ does not increase from one step no another. Hence, the convergence of the algorithm is always ensured.

It should be pointed out that, depending on the initialization of $\Lambda$, the algorithm will converge to a different suboptimal 
solution. Moreover, it can happen that for a fixed $\Lambda$, step 1 has no feasible solution. On the other hand, it can be observed that for $\Lambda=0$, step 1 will always present a feasible solution. That one corresponds to the case where the set of asymptotic stability is contained in the region of linearity of the closed-loop system. Hence, a recommended first initial guess for $\Lambda$, always feasible, would be the matrix 0 .

The solution of the optimal problems in steps 1 and 2 involves the solution of LMIs. Nowadays, considering systems of reasonable dimensions, we can say that these problems can be efficiently solved by means of commercial and free packages (LMI solvers). Of course, large scale problems can suffer from numerical unstability. Concerning, the processing-time, it will depend on the dimension of the considered system. However, even it is large, this is not a problem because the computations are made off-line.

\subsection{Gain Constraints}

A constraint of anti-windup gain limitation can be added to the optimization problem (23) as follows.

Note that, since

$$
E_{c}=Z S^{-1}
$$

it follows that $E_{c(i, j)}=Z_{(i, j)} S_{(j, j)}^{-1}$

Hence, if

$$
\left[\begin{array}{cc}
S_{(j, j)} \sigma & Z_{(i, j)} \\
Z_{(i, j)} & S_{(j, j)}
\end{array}\right] \geq 0
$$

by the Schur's complement one has

$$
\sigma-Z_{(i, j)} S_{(j, j)}^{-1} Z_{(i, j)} S_{(j, j)}^{-1} \geq 0
$$

which ensures that $\left(E_{c(i, j)}\right)^{2} \leq \sigma$

By the same reasoning, structural constraints on $E_{c}$ can be take into account in (23) by fixing some of the elements of matrix $Z_{(j, i)}$ as zero.

\section{ILLUSTRATIVE EXAMPLE}

Consider the following linear open-loop unstable system:

$$
\begin{aligned}
& x(t+1)=1.2 x(t)+u(t) \\
& y(t)=x(t)
\end{aligned}
$$

and the stabilizing PI controller

$$
\begin{aligned}
& x_{c}(t+1)=x_{c}(t)-0.05 y(t) \\
& v_{c}(t)=x_{c}(t)-y(t) \\
& u(t)=\operatorname{sat}\left(v_{c}(t)\right)
\end{aligned}
$$

Let the shape set $\Xi_{0}$ be defined by as a square region in the space $\Re^{2}$ :

$$
\Xi_{0}=\operatorname{Co}\left\{\left[\begin{array}{l}
1 \\
1
\end{array}\right] ;\left[\begin{array}{c}
1 \\
-1
\end{array}\right] ;\left[\begin{array}{c}
-1 \\
1
\end{array}\right] ;\left[\begin{array}{l}
-1 \\
-1
\end{array}\right]\right\}
$$

Considering, the control bound $u_{0}=1$ and a scaling factor $\beta$ we aim to compute an anti-windup gain $E_{c}$ in order to obtain a region of stability $\beta \Xi_{0} \subset \mathcal{E}\left(P, \gamma^{-1}\right)$ with $\beta$ as large as possible.

Using Algorithm 1, the obtained optimal solution is $\beta=$ 1.5729 with:

$$
\begin{gathered}
\Lambda=0.756 ; \quad P=\left[\begin{array}{rr}
0.0613 & -0.0405 \\
-0.0405 & 0.2619
\end{array}\right] \\
\gamma=1 ; \quad E_{c}=-0.0011
\end{gathered}
$$

The obtained domain of stability is shown in figure 2 . The figure also depicts several trajectories of the closed-loop system as an attempt to illustrate its basin of attraction. Regarding the state of the plant, it can be seen that the domain of stability is confined to the interval $x(0) \in(-5,5)$. In fact, for $x(0)=5$ and $x_{c}(0) \in(-\infty, 4]$, the state of the controller diverges towards $-\infty$ while the state of the plant remains at its initial value (a similar reasoning holds for the symmetric trajectory). The ellipsoidal estimate of the domain of stability is seen to span beyond the region of linearity meaning that saturation does effectively occur for certain initial conditions inside the estimated domain of stability. On the other hand, the ellipsoidal estimate includes points that are close to the boundaries of the basin of attraction, especially in the direction of the state of the plant, thus providing a reasonable estimate of the basin of attraction. In this regard, it is important to remark that the optimization criterion and the choice of $\Xi_{0}$ are degrees of freedom that influence the ellipsoidal estimate of the basin of attraction.

\section{CONCLUDING REMARKS}

We have provided a method to design an anti-windup gain aiming at enlarging the region of asymptotic stability of linear control systems with saturated inputs. The method considers a given output linear feedback designed for the original systems in the absence of saturation, and provide a design of an anti-windup gain in order to improve an estimate of its region of asymptotic stability. Such an improvement is always possible since the trivial solution (zero gain) is part of the set of solutions encompassed by the method.

The numerical algorithms proposed simplify considerably in the single-input case. In this case, the optimal solution can be found by a simple griding procedure. In general, the pro- 


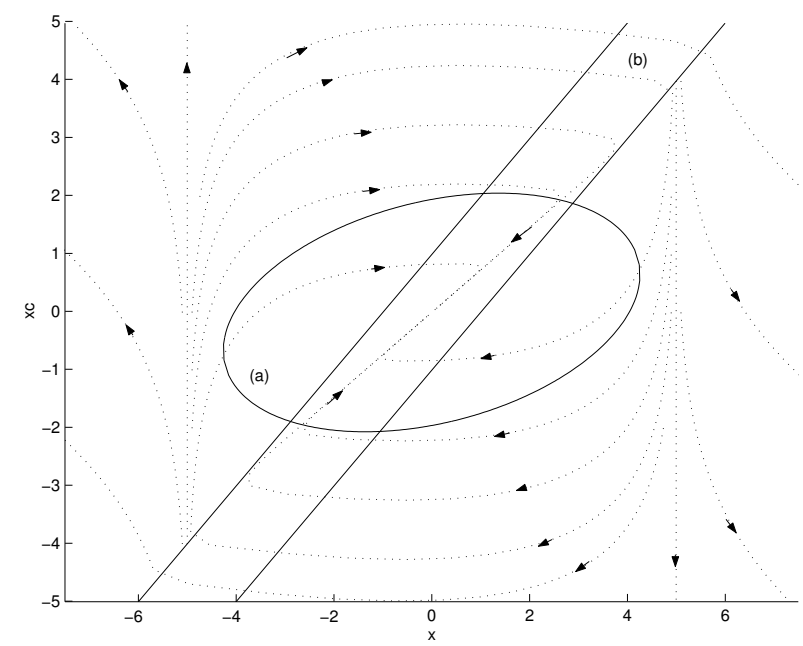

Figure 2: (a) obtained region of stability ; (b) region of linearity

posed methods involve solving BMIs, which can be accomplished by means of relaxation algorithms presented in the paper.

\section{REFERENCES}

Åström, K. J. and Rundqwist, L. (1989). Integrator windup and how to avoid it, American Control Conference, Pittsburgh, PA, pp. 1693-1698.

Barbu, C., Reginatto, R., Teel, A. R. and Zaccarian, L. (2000). Anti-windup for exponentially unstable linear systems with inputs limited in magnitude and rate, American Control Conference, Chicago, IL.

Boyd, S., El Ghaoui, L., Feron, E. and Balakrishnan, V. (1994). Linear Matrix Inequalities in System and Control Theory, SIAM Studies in Applied Mathematics.

Burgat, C. and Tarbouriech, S. (1998). Intelligent antiwindup for systems with input magnitude saturation, Int. J. Robust Nonlinear Control 8: 1085-1100.

Fertik, H. A. and Ross, C. W. (1967). Direct digital control algorithm with anti-windup feature, ISA Transactions 6: $317-328$.

Gomes da Silva Jr., J. M., Paim, C. and Castelan, E. (2001). Stability and stabilization of linear discrete-time systems subject to control saturation, 1st IFAC Symposium on System Structure and Control, Prague, Czech Republic.

Gomes da Silva Jr., J. M., Tarbouriech, S. and Reginatto, R. (2002). Analysis of regions of stability for linear systems with saturating inputs trhough an anti- windup scheme, IEEE-Conference on Control Applications 2002, Glasgow, UK.

Kapoor, N., Teel, A. R. and Daoutidis, P. (1998). An antiwindup design for linear systems with input saturation, Automatica 34(5): 559-574.

Khalil., H. K. (1992). Nonlinear Systems, MacMillan.

Kothare, M. V. and Morari, M. (1997). Stability analysis of anti-windup control scheme: a review and some generalizations, European Control Conference, Brussels, Belgium.

Kothare, M. V. and Morari, M. (1999). Multiplier theory for stability analisys of anti-windup control systems, Automatica 35: 917-928.

Miyamoto, A. and Vinnicombe, G. (1996). Robust control of plants with saturation nonlinearity based on coprime factor representation, Conference on Decision and Control, Kobe, Japan, pp. 2838-2840.

Shamma, J. S. (1999). Anti-windup via constrained regulation with observers, American Control Conference, San Diego, USA, pp. 2481-2485.

Teel, A. R. (1999). Anti-windup for exponentially unstable linear systems, Int. J. Robust Nonlinear Control 9(10): 701-716.

Teel, A. R. and Kapoor, N. (1997). The $\mathcal{L}_{2}$ anti-windup problem: Its definition and solution, European Control Conference, Brussels, Belgium.

Walgama, K. S. and Sternby, J. (1993). Conditioning technique for multiinput multioutput processes with input saturation, IEE Proc. D 140(4): 231-241. 